\title{
Properties of the barium strontium titanate film on the silicon substrate
}

\author{
R.I. Batalov ${ }^{1}$, D.K. Zharkov ${ }^{1}$, D.P. Pavlov ${ }^{1}$, S.A. Migachev ${ }^{1}$, I.V. Lunev ${ }^{2}$, A.S. Elshin ${ }^{3}$,
} A.V. Leontyev ${ }^{1}$, A.O. Chibirev ${ }^{2}$, T.S. Shaposhnikova ${ }^{1}, \underline{\text { R.F. Mamin }}^{1,2}$

${ }^{I}$ Zavoisky Physical-Technical Institute, FRC Kazan Scientific Center of RAS, 420029, Kazan, Russia dmitry.p.pavlov@gmail.com

${ }^{2}$ Institute of Physics, Kazan Federal University, Kazan 420008, Russia

${ }^{3}$ Moscow Technological University, 191454, Moscow, Russia

To improve the properties of solar cells, primarily to increase the absorptivity of solar cells in a wider region of the light spectrum [1], various films are applied to their surface. Also recently, the photovoltaic effect is often used [2], which is the direct conversion of light into electricity and is considered one of the most reliable and abundant sources of renewable and clean energy. A high-mobility electron gas was observed in 2004 [3] at the interface of $\mathrm{LaAlO}_{3} / \mathrm{SrTiO}_{3}$ heterostructure. In this work ee solve these problems by creating such a special state of the interface when applying a thin ferroelectric film on silicon. The properties of barium-strontium titanate (BST) films on a silicon substrate and the possibility of their modification are investigated. The properties of the heterostructures obtained by dielectric and various optical methods are measured and characterized. It was found that changes of the capacity of the heterostructure are related to increase of conductivity through the Si layer with increasing frequency based on the results of the study of the frequency dependences of the BST/Si heterostructure. The reflection spectrum from the BST film was studied. Based on these results, the absorption spectrum was modeled, which has a peak in the region of $3 \mathrm{eV}$. There is also an inflection in the region of 2.6$2.8 \mathrm{eV}$, which can correspond to energy from the top of the valence band to the lower levels of the BST film defect system. The photoconductivity of the heterostructure interface and the possibility of modifying the properties of barium-strontium titanate films by the laser annealing method and the ion implantation method are studied. The surface was tested by atomic force microscopy before and after modification. The laser annealing method did not yield any significant results. The most promising method was the modification of barium-strontium titanate films on a silicon substrate by the ion implantation method with the introduction of chromium atoms with energy of $40 \mathrm{keV}$. The effect of modification on photoconductive properties of the films was revealed. The measurement of the photoconductivity has shown that current at the interface increases substantially when illuminated by the ultraviolet light. This increase is particularly high for samples modified by ion implantation. The possibility of carrier concentration increasing by this way is discussed when exciting the $p / n$ junction for use as an operating element in solar batteries.

The reported study was supported of the Russian Foundation for Basic Research, research project No. 18-42-160005. The work is partially performed according to the Russian Government Program of Competitive Growth of Kazan Federal University. A.S. Elshin thanks the Russian Foundation for Basic Research for financial support, project No. 17-32-50047.

1. Ch.S. Solankii, H.K. Singh, Anti-refrection and Light Trapping in c-Si Solar Cells. (Singapore, Springer) 2: 186 p. (2017).

2. S.Y. Yang, J. Seidel, S.J. Byrnes, P. Shafer, C.-H. Yang, M.D. Rossell, P. Yu, Y.-H. Chu, J.F. Scott, J.W. Ager III, L.W. Martin, R. Ramesh, Nature Nanotechnology 5, 143 (2010).

3. A. Ohtomo, and H. Hwang, Nature 427, 6973 (2004). 